\title{
PERCEPCIÓN DE LA CALIDAD DE VIDA Y LA SALUD MENTAL EN OFICIALES DE LA FUERZA AÉREA COLOMBIANA
}

\author{
Luis Artemo González-Quevedo, Psicólogo ${ }^{1 *}$, Pablo Alfonso Sanabria-Ferrand, Psicólogo M. Sc. ${ }^{1}$ \\ y T.C. Humberto Zuluaga MD. Psiquiatra ${ }^{2}$ \\ ${ }^{1}$ Grupo de Investigación Salud y Comportamiento, Facultad de Medicina, Universidad Militar Nueva \\ Granada, Bogotá. ${ }^{2}$ Centro de Medicina Aeroespacial - Fuerza Aérea Colombiana, Bogotá
}

\begin{abstract}
Resumen
Debido a la situación de orden público interno en Colombia los oficiales de la Fuerza Aérea Colombiana (FAC) están expuestos a situaciones estresantes potencialmente traumáticas. El objetivo del estudio fue determinar la percepción de calidad de vida de oficiales y su relación con factores de la salud mental con el fin de aportar elementos de discusión para el fortalecimiento de programas de promoción y prevención. Se utilizó una metodología correlacional tipo encuesta de prevalencia, la muestra fue de 296 oficiales de vuelo voluntarios y 200 oficiales de otras especialidades de la FAC en todo el País. Se evaluaron cinco aspectos de calidad de vida general (autoaceptación, adaptación al medio, estabilidad socioafectiva, integridad física y proyección), tres de calidad de vida militar (reconocimiento, apoyo institucional y cohesión) y seis indicadores de psico-patología (depresión, ansiedad, manía, psicosis, estrés postraumático y trastorno disocial). Todas las escalas fueron calibradas con base en la teoría de respuesta al item. Los resultados mostraron niveles de calidad de vida general y militar altos y que los niveles bajos se asocian con altas probabilidades de tener alguna psicopatología. Los oficiales de vuelo tuvieron niveles similares de calidad de vida comparados con oficiales de de otras especialidades excepto en proyección en la que fueron inferiores. Los resultados sugieren que es necesario intervenir diferentes aspectos (sociales, institucionales, personales, entre otros) para mejorar los niveles de calidad de vida y disminuir la probabilidad de la presencia de psicopatología.
\end{abstract}

Palabras clave: salud mental, calidad de vida, personal militar, Colombia

\section{PERCEPTION OF QUALITY OF LIFE AND MENTAL HEALTH IN OFFICERS OF THE COLOMBIAN AIR FORCE}

\begin{abstract}
Due to internal public order situation in Colombia, officials of the Colombian Air Force (FAC) are exposed to potentially traumatic stressors. The objective of this study was to determine officials' perception of their quality of life and its relationship with mental health in order to provide elements for discussion for the development of promotion and prevention programs. A correlational methodology was used; the sample was of 296 volunteer flight officers and 200 FAC non-flying officers from different locations from Colombia. Five aspects of quality of life (self-acceptance, adaptation, socio-affective stability, physical integrity and personal projection), three aspects of the quality of military life (recognition, institutional support and cohesion) and 6 indicators of psychopathology (depression, anxiety, mania,
\end{abstract}

* Correspondencia: Luis Artemo González luis.gonzalezq@unimilitar.edu.co. Dirección Correspondencia: Tr. 5 No 49-00, Facultad de Medicina, Universidad Militar Nueva Granada, Bogotá, Colombia.

Recibido: Agosto 2 de 2009 Aceptado: Septiembre 16 de 2009. 
psychosis and post-traumatic stress disorder) were evaluated. All scales were calibrated based on the item response theory. The results showed high levels of overall life quality and military life quality and that low levels are associated with higher probabilities of having any psychopathology. Flight officers had similar levels of quality of life compared with ground officers except on the projection aspect, which was lower. Results suggest there is a need to work in various aspects (social, institutional, personal) to improve levels of quality of life and lower levels of psychopathology.

Keywords: military personnel, quality of life, mental health, pilots, colombian

\title{
PERCEPÇÃO DE QUALIDADE DE VIDA E SAÚDE MENTAL DE TODO OFICIAIS DA FORÇA AÉREA COLOMBIANA
}

\begin{abstract}
Resumo
Devido à situação de ordem pública interna na Colômbia todo oficiais de Força Aérea Colombiana (FAC) estão exposto a fatores estressantes potencialmente traumática. O objetivo deste estudo foi determinar a percepção de qualidade de vida do oficial e sua relação com a saúde mental a fim de fornecer elementos de discussão para o desenvolvimento de programas de promoção e prevenção. Foi utilizada a metodologìa correlacional tipo inquérito de prevalência, a amostra foi de 296 voluntàrios e 200 indivìduos de outras especialidades da FAC em todo o país. Foram avaliados cinco aspectos da qualidade de vida (auto-aceitação, adaptação ambiental, a estabilidade sócio-afetiva, física e projeção), três da qualidade de vida militar (reconhecimento, apoio institucional e de coesão) e seis indicadores de psicopatologia (depressão, ansiedade, psicose, mania, transtorno de estresse pós-traumático e transtorno disocial). Todas as escalas foram calibrados com base na teoria de resposta ao item. Os resultados mostraram níveis de qualidade de vida global e nìvel militar alto. Os níveis baixos estão associados a maior chance de ter alguma psicopatologia. A tripulação do ar tinham níveis semelhantes de qualidade de vida em comparação com os oficiais de terra; só um item foi menor: a projeção. Os resultados sugerem a necessidade de intervir em vários aspectos (social, institucional, etc) para melhorar os níveis de qualidade de vida e abaixar a presença de psicopatologia.
\end{abstract}

Palavras-chave: saúde mental, qualidade de vida, militares, Colômbia

\section{Introducción}

Existe bastante evidencia científica del impacto que tiene el estrés en el trabajo sobre la salud y la calidad de vida. Ese impacto está mediado no sólo por las potencialidades individuales, sino también por la intensidad y el tiempo de exposición a la situación de estrés.

La vida militar hace que las personas estén expuestas más frecuentemente a situaciones potencialmente traumáticas y a un ambiente de trabajo constantemente estresante, situación que se agudiza si se participa en conflictos armados o guerras. Esto ha llevado a muchos investigadores, especialmente de países que tienen ejércitos en situación de guerra como Estados Unidos e Inglaterra, a realizar estudios que indaguen sobre los efectos del trabajo como militar en la vida de las personas. A partir de la Guerra del Golfo (1990-1991) se incrementaron los estudios con veteranos de Estados Unidos. Algunos estudios evaluaron el impacto de la experiencia de la Guerra del Golfo sobre la salud física (1-3), mortalidad $(4 ; 5)$ y salud mental en general (6), estrés postraumático (7-9), ansiedad (10) y trastornos psiquiátricos $(11,12)$. Por su parte, los ingleses han desarrollado varios estudios con veteranos en salud física (13-15), salud mental $(16 ; 17)$ y en riesgo ocupacional $(18,19)$. También existen trabajos con ejércitos alemanes $(20)$ y australianos $(21,22)$ en temas relacionados con salud mental.

Con personal activo, igualmente, se han realizado varios estudios que abordan diferentes aspectos de la salud del personal militar tales como trastornos psiquiátricos $(12,23)$, mortalidad por lesiones (24), estrés ocupacional $(25,26)$, salud mental $(27-32)$, 
estrés postraumático (33-35), consumo de sustancias $(36,37)$ y dependencia a la nicotina (38). Todos estos estudios muestran el interés de los investigadores por la población militar, dado su alto riesgo de tener algún problema médico o de salud mental, y con el fin de tener elementos de intervención preventivos para disminuir el impacto negativo en estas personas.

Aunque en Colombia no se puede hablar de una guerra regular como la del Golfo, si existe una situación de confrontación que podría ser comparable en cuanto los niveles de estrés que produce al personal militar involucrado en esta situación. Sin embargo, no se han realizado muchos estudios que midan el impacto en la salud física, la salud mental, la situación psicosocial en personal militar retirado o activo que haya estado durante mucho tiempo en situaciones de combate. A nivel de salud física no se conoce ningún estudio que abarque una gran población; en salud mental, en 1997 se realizó por primera vez un estudio en Fuerzas Militares (39), que incluyó al Ejercito, la Armada y la Fuerza Aérea y que arrojó el primer diagnóstico de salud mental y consumo de sustancias psicoactivas en personal activo. En el 2007, se realizó un estudio de calidad de vida y salud mental pero únicamente en la Fuerza Aérea Colombiana (40). Estos estudios han permitido a las Fuerzas Militares tener una aproximación a la situación en salud mental del personal activo.

Un aspecto importante menos mencionado o mencionado indirectamente en los estudios americanos $e$ ingleses es el de la calidad de vida. El concepto de calidad de vida se ha estudiado desde una perspectiva biomédica, psicológica y social, y se ha definido, principalmente desde un punto subjetivo, como la percepción de las personas sobre diversos ámbitos de sus vidas, destacando la relevancia del contexto social, cultural y del sistema de valores en el que experimentan su vida cotidiana (41).

Los estudios realizados en este campo con veteranos de la guerra del Golfo $(42,43)$ hacen énfasis en los aspectos biomédicos de la calidad de vida, especialmente lo relacionado con la salud, utilizando el cuestionario SF-36 como instrumento de medición; este cuestionario mide ocho dimensiones que son: función física, rol físico, dolor corporal, salud general, vitalidad, función social, rol emocional y salud mental.

Sin embargo, estos estudios no abordan una evaluación más amplia de la calidad de vida tal como la establece la Organización Mundial de la Salud (OMS) (44), la cual define aspectos tales como el psicológico, las relaciones sociales, los niveles de independencia, la relación con el medio ambiente, y la espiritualidad o religiosidad como determinantes de la calidad de vida general de la personas. Aunado a esta visión más amplia de la calidad de vida, se ha desarrollado el concepto de la salud positiva, que a diferencia del enfoque convencional que investiga sobre las causas generales de las enfermedades, se centra en estudiar como permanece el hombre sano.

Para el caso de salud mental, igualmente, se ha dedicado mucho esfuerzo a estudiar los aspectos negativos y patológicos del ser humano (ansiedad, estrés, depresión, etc), dejando de lado a menudo el estudio de aspectos más positivos como, por ejemplo, la creatividad, la inteligencia emocional, el humor, la sabiduría, la felicidad, la resiliencia, etc. (45).

Para este trabajo se asumió que la salud mental es un continuo que va desde estar sano hasta tener alguna patología, tal y como lo propone Aponte y col. (46) al plantear que la calidad de vida y la salud mental hacen parte de un continuo y no se puede plantear una como causa de otro. En este continuo los aspectos relacionados con estar sano (aspectos positivos) se definieron como calidad de vida y los aspectos negativos se definieron como patologías. Se definieron cinco factores básicos de la calidad de vida similares a los que se plantean en las escalas de bienestar psicológico $(47,48)$ : la autoaceptación, la adaptación al medio, la estabilidad socioafectiva, la integridad física y la proyección. Para el caso de las patologías, se definieron las más prevalentes y tal como las define el Diagnostic and Statistical Manual of Mental Disorders (DSM IV), que es una herramienta de diagnóstico. Los instrumentos utilizados tanto para medir la calidad de vida como las psicopatologías están calibrados bajo el modelo Rash de la Teoría de Respuesta al Ítem (49). Con este modelo de medición se puede determinar la probabilidad que tiene una persona de presentar el evento evaluado a través del cuestionario; en otras palabras el puntaje obtenido por cada sujeto indica el riesgo de tener el evento medido.

Medir los aspectos "positivos" y "negativos" de la salud mental permitió:

1. Tener una visión más amplia de la salud mental en el personal de oficiales. 
2. Aportar mejores elementos para realizar intervenciones preventivas a un nivel masivo y con mayores probabilidades de éxito puesto que la mayoría de los estudios poblacionales de salud mental, sólo evalùan las psicopatologías sin aportar muchos elementos de intervención diferentes al incremento del número de psiquiatras y psicólogos contratados.

3. Aportar elementos de análisis que apoyen o rechacen la hipótesis de que la calidad de vida y las patologías hacen parte de un continuo desde estar sano hasta enfermarse.

Se propuso en este trabajo determinar la percepción de los oficiales de la FAC sobre su calidad de vida y establecer su relación con los factores de salud mental (psicopatologías) con el fin de aportar elementos científicos para los programas de promoción y prevención que tiene la Fuerza Aérea.

\section{Método}

\section{Tipo de estudio}

Se hizo un estudio correlacional con base en la información obtenida del estudio: "Calidad de vida y salud mental en la Fuerza Aérea Colombiana" (40).

\section{Muestra}

La muestra estuvo conformada por 296 oficiales de vuelo y 200 oficiales de otras especialidades de la FAC, seleccionados al azar de todas las unidades, y que voluntariamente decidieron participar en el estudio. El diez por ciento fueron mujeres y la media del tiempo de servicio fue de 11,4 años con $\mathrm{SD}=5,2$.

\section{Instrumentos}

Se utilizó la encuesta calidad de vida y salud mental propuesta en el estudio "Condiciones teórico-metodológicas para la realización del diagnóstico de salud mental en Bogotá" (46), adaptada a población militar. Esta permitió obtener información sobre los siguientes aspectos:

\section{Calidad de vida}

La encuesta permitió medir la percepción de calidad de vida a través de cinco ejes o factores: autoaceptación, aceptación al medio, estabilidad socioafectiva, integridad física y proyección.
Autoaceptación: hace referencia al conocimiento que tiene la persona de sí mismo; es un estado de madurez que se logra cuando la persona tiene una comprensión profunda de sí, de sus fortalezas y debilidades, de sus defectos y virtudes en todas las dimensiones de su ser. Adaptación al medio: mide las habilidades cognitivas y afectivas necesarias para que una persona pueda ajustarse al medio en el que se desarrolla y mas aún, pueda llegar a transformarlo para su bienestar y el de los demás. Estabilidad socioafectiva: hace referencia a las acciones y actitudes que dan cuenta de la solidez y satisfacción en las relaciones interpersonales en distintos contextos. Integridad física: hace referencia a situaciones que permitan evidenciar calidad de vida en relación con la satisfacción de necesidades básicas como la alimentación, la seguridad y la salud física. Proyección: este eje establece las situaciones de oportunidad que brinda el medio a la persona para superar el simple nivel de sobrevivencia y acceder a niveles elevados de actualización, preparación, formación, disfrute, etc.

Psicopatologías

La encuesta permite establecer cuáles personas tienen síntomas y/o conductas que indican una alta probabilidad de tener alguna de las siguientes patologías: estrés postraumático, ansiedad, depresión, manía, trastorno disocial y psicosis.

\section{Calidad de vida militar}

Se elaboró y se validó un instrumento específico de calidad de vida militar ya que el instrumento de calidad de vida general no incluía ciertos aspectos que son propios de la vida militar. Por medio del análisis factorial se determinaron tres factores: reconocimiento, se refiere a incentivos mediados por los superiores directos, tales como felicitaciones, condecoraciones, traslados, comisiones, etc.; apoyo institucional, que hace referencia a las condiciones institucionales que no requieren de intermediación de superiores tales como salario, sistema de salud, educación continuada, etc. y cohesión, hace relación a la confianza percibida por parte de sus superiores, compañeros y subalternos, así como el reconocimiento por estos de su labor dentro de la institución. Todas las escalas incluidas en la encuesta fueron calibradas bajo la teoría de respuesta al ítem (TRI)(49). 


\section{Procedimiento}

Se accedió a la base de datos del estudio mencionado tomando la información de todos los oficiales; luego por medio del software MULTILOG y con base en el modelo de Rash o de un parámetro, se obtuvo el logit que indicaba la probabilidad de tener el evento medido. Luego se clasificaron a las personas en si tenían o no el evento medido asumiendo como corte tener una probabilidad de $75 \%$ o más. Dado que el dato es una probabilidad, hace más robusta la medición de los diferentes aspectos medidos en la encuesta. Finalmente se obtuvieron los índices de correlación Pearson.

\section{Resultados}

Los valores altos de probabilidad asociados a los logits obtenidos de la calificación de los instrumentos indican una mayor probabilidad de tener la dimensión medida. Para el caso de calidad de vida un valor alto indica que tiene la probabilidad de tener un déficit en la dimensión evaluada.

Con respecto a las escalas de calidad de vida general se encontró que un $35 \%$ de los oficiales tienen al menos un factor insatisfactorio y es básicamente igual para los oficiales de vuelo que para los oficiales de otras especialidades. También se observó que el factor donde se encontró un valor más alto fue en estabilidad socioafectiva (15\%) y el más bajo fue la autoaceptación (9\%).
Respecto a la calidad de vida militar se encontró que en cada uno de los factores (reconocimientos, apoyo institucional y cohesión) hay un $11 \%$ de oficiales insatisfechos, y en los oficiales de vuelo los valores son ligeramente más altos pero no estadísticamente diferentes.

La tabla 1 muestra que la ansiedad y la depresión son las patologías que se correlacionan de forma positiva con casi todos los factores de calidad de vida; es decir, que un puntaje alto en insatisfacción en los factores de calidad de vida está acompañado con puntuaciones altas en riesgo de presentar patología. El trastorno disocial solamente correlaciona con autoaceptación. Igualmente se observó que la estabilidad socioafectiva no correlacionó con ninguna probabilidad de presencia de psicopatología.

En la tabla 2 se observa que los factores de calidad de vida militar correlacionan con todos las probabilidades de presencia de psicopatologías, excepto trastorno disocial y manía. También se evidencian valores más altos en comparación con los de calidad de vida general de la tabla 1 . Los valores más altos están en el riesgo de patología de ansiedad y depresión, igualmente el factor apoyo institucional es el factor que tiene correlaciones más altas con las patologías. Con respecto al trastorno de estrés postraumático no se encontró correlación con ningún factor de calidad de vida general ni militar, ya que el riesgo de presentar este trastorno fue muy bajo.

TABLA 1. Correlación de los factores de calidad de vida general y los factores de salud mental.

\begin{tabular}{|c|c|c|c|c|c|c|}
\hline & & Manía & Ansiedad & $\begin{array}{l}\text { Trastorno } \\
\text { Disocial }\end{array}$ & Depresión & Psicosis \\
\hline \multirow{2}{*}{ Autoaceptación } & $\mathrm{rp}$ & $0,17 * *$ & $0,16^{*}$ & $0,20 * *$ & $0,15^{*}$ & $0,16^{*}$ \\
\hline & $\mathrm{N}$ & 291 & 291 & 287 & 291 & 291 \\
\hline \multirow{2}{*}{$\begin{array}{l}\text { Adaptación al } \\
\text { medio }\end{array}$} & $\mathrm{rp}$ & & $0,15^{*}$ & & $0,16^{*}$ & \\
\hline & $\mathrm{N}$ & & 290 & & 290 & \\
\hline \multirow{2}{*}{ Integridad física } & $\mathrm{rp}$ & $0,14^{*}$ & $0,15^{*}$ & & $0,21 * *$ & \\
\hline & $\mathrm{N}$ & 290 & 290 & & 290 & \\
\hline \multirow{2}{*}{ Proyección } & $\mathrm{rp}$ & & & & $0,13^{*}$ & \\
\hline & $\mathrm{N}$ & & & & 284 & \\
\hline
\end{tabular}

* Significativo a 0,05

** Significativo a 0,01 
TABLA 2. Correlación de los factores de calidad de vida militar y los factores de salud mental

\begin{tabular}{lcccccc}
\hline & & Ansiedad & $\begin{array}{c}\text { Trastorno } \\
\text { Disocial }\end{array}$ & Depresión & Psicosis & Mania \\
\hline \multirow{2}{*}{ Reconocimiento } & $\mathrm{rp}$ & $0,29 * *$ & $0,20^{* *}$ & $0,30^{* *}$ & $0,29 * *$ & $0,18^{* * *}$ \\
& $\mathrm{~N}$ & 286 & 280 & 110 & 285 & 284 \\
Apoyo institucional & $\mathrm{rp}$ & $0,32^{* *}$ & $0,24 * *$ & $0,36^{* *}$ & $0,27^{* *}$ & $0,22^{* *}$ \\
& $\mathrm{~N}$ & 286 & 280 & 110 & 285 & 284 \\
Cohesión & $\mathrm{rp}$ & $0,25^{* *}$ & & $0,23^{* *}$ & $0,22^{* *}$ & $0,21^{* *}$ \\
& $\mathrm{~N}$ & 286 & & 110 & 285 & 284 \\
\hline
\end{tabular}

* Significativo a 0,05

** Significativo a 0,01

\section{Discusión}

La revisión de los diferentes estudios en personal militar permitió ver el gran interés de los investigadores en los diferentes países, por establecer el impacto de la vida militar, especialmente en situación de guerra, sobre la calidad vida de estas personas. Estos trabajos han demostrado que por ejemplo el personal militar que ha estado en situación de guerra tiene más riesgo de tener problemas de salud física $(1,33)$ y de salud mental $(10,16,25,50,51)$. Con respecto a calidad de vida se ha encontrado que en general es más pobre $(42,43)$.

Esto lleva a pensar que si en Colombia se tiene una situación de orden público interno tan prolongada, el impacto en el personal militar podría ser comparable con el que ha provocado en otros países, aunque se desconocen estudios específicos en pilotos. Esto hace plantear la necesidad urgente de abordar estudios en los diferentes aspectos de la vida de estas personas (salud física, salud mental, aspectos psicosociales, ocupacionales, calidad de vida, etc.), que permitan adoptar medidas no sólo desde el punto de vista de la salud sino también en el social y el económico.

Los datos de prevalencia de los factores de calidad de vida no son altos (entre $8 \%$ y $15 \%$ ), es decir que los oficiales tienen una buena calidad de vida general; sin embargo, es importante fortalecer programas de bienestar y salud para reducir estos porcentajes. A pesar de la información obtenida, estas cifras no se pueden comparar con otras poblaciones civiles o militares, debido a la metodología y los instrumentos utilizados; sin embargo, motiva a que se hagan estudios que utilicen otros instrumentos que permitan evaluar la evolución de la situación de calidad de vida y comparar los resultados con otras poblaciones.

Con respecto a la calidad de vida militar igualmente no existe una referencia para comparar los resultados. Para el caso de las psicopatologías, aunque existen estudios nacionales e internacionales en poblaciones civiles y militares que establecen las diferentes prevalencias, dado el enfoque metodológico y de instrumentos, estas cifras no se pueden comparar. Aunque pareciera una limitante del trabajo no tener cifras comparables con estudios actuales, quizá es una fortaleza porque se propone una forma diferente de instrumentos con un amplio y actualizado desarrollo psicométrico basado en la Teoría de respuesta al Ítem (49), superando los enfoques clásicos de la medición.

Los niveles bajos de calidad de vida general y militar se asociaron con una mayor probabilidad de presentar psicopatologías y esta correlación es más fuerte con los factores de calidad de vida militar, hallazgo que apoya la hipótesis planteada por Aponte (46), que la calidad de vida y la salud mental hacen parte de un continuo. De igual forma, estos datos permiten plantear alternativas, como mejorar los factores de calidad de vida, para prevenir la presencia de psicopatologías. Una intervención en estos factores es menos costosa y mucho más fácil de llegar a todos las personas, pues se puede lograr mejorando las políticas globales de bienestar, de ambiente de trabajo y con talleres grupales de intervención. Si se observa la salud mental desde una perspectiva tradicional, requeriría el em- 
pleo de muchos psiquiatras y psicólogos para hacer intervenciones individuales, lo cual sería mucho más costoso. Los programas con una orientación positiva de la salud mental tienen una ventaja adicional, que es facilitar el acceso de algunas personas, que no van a donde un psiquiatra o psicólogo por miedo a una estigmatización como "enfermo mental".

Las correlaciones observadas entre la calidad de vida militar y calidad de vida general con las psicopatologías indica que la presencia de psicopatología depende de muchos factores (sociales, institucionales, personales, etc.) y que por lo tanto las intervenciones se deben dar en todos los niveles, si se quiere mejorar el estado de salud mental. Se requieren unas políticas institucionales y un compromiso en todos los niveles de mando así como del personal del área de salud mental, para favorecer las condiciones que mejoren la calidad de vida y propendan por la salud mental de los integrantes de la institución militar.

Igualmente, estos resultados deben servir para evaluar, redireccionar y focalizar las diferentes políticas y programas de bienestar y de promoción y prevención en salud mental que tiene actualmente la Fuerza Aérea, con el fin de evitar la presencia de psicopatologías en el personal, las cuales tendrían un alto costo para el individuo y para la Fuerza. Finalmente se espera que este trabajo estimule el interés de la Fuerzas Militares y de los investigadores para incrementar el número de trabajos científicos en la población militar

\section{Agradecimientos}

Los autores agradecen a la Universidad Militar Nueva Granada por la financiación del proyecto MED 2005-02 y a la Fuerza Aérea Colombiana a través de su Centro de Medicina Aeroespacial por su participación activa durante todo el proyecto.

\section{Referencias}

1. Fukuda K, Nisenbaum R, Stewart G, Thompson W W, Robin L, Washko R M, et al. Chronic Multisymptom Illness affecting Air Force Veterans of the Gulf War. JAMA. 1998;280(11):981-8.

2. Smith B, Smith T C, Ryan M A, Gray G C. A Comparison of the Postdeployment Hospitalization Experience of U.S. Military Personnel following Service in the 1991 Gulf War, Southwest Asia after the Gulf War, and Bosnia. Journal of Occupational and Environmental Hygiene. 2006;3(12):660-70.

3. Smith B, Chu L K, Smith T C, Amoroso P J, Boyko E J, Hooper T I, et al. Challenges of Self-reported Medical Conditions and Electronic Medical Records among Members of a Large Military Cohort. BMC Medical Research Methodology. 2008;8:37.
4. Kang H K, Bullman T A. Mortality among U.S. Veterans of the Persian Gulf War. New England Journal of Medicine. 1996;335(20):1498-504.

5. Kang H K, Bullman T A. Mortality among US Veterans of the Persian Gulf War: 7-Year Follow-up. American Journal of Epidemiology. 2001;154(5):399-405.

6. O'Donnell J C. Military Service and Mental Health in Later Life. Military Medicine. 2000;165(3):219-23.

7. Barrett D H, Doebbeling C C, Schwartz D A, Voelker M D, Falter K H, Woolson R F, et al. Posttraumatic Stress Disorder and Self-reported physical Health Status among U.S. Military Personnel serving during the Gulf War period: A Populationbased Study. Psychosomatic6s. 2002;43(3):195-205.

8. Tuerk P W, Grubaugh A L, Hamner M B, Foa E B. Diagnosis and Treatment of PTSD-related Compulsive checking Behaviors in Veterans of the Iraq War: The Influence of Military Context on the Expression of PTSD Symptoms. American Journal of Psychiatry. 2009;166(7):762-7.

9. Spiro A 3rd, Hankin C S, Mansell D, Kazis L E. Posttraumatic Stress Disorder and Health Status: The Veterans Health Study. Journal of Ambulatory Care Managent. 2006;29(1):71-86.

10. Black D W, Carney C P, Peloso P M, Woolson R F, Schwartz D A, Voelker M D, et al. Gulf War Veterans with Anxiety: Prevalence, Comorbidity, and Risk Factors. Epidemiology. 2004;15(2):135-42.

11. Stimpson N J, Thomas H V, Weightman A L, Dunstan F, Lewis G. Psychiatric Disorder in Veterans of the Persian Gulf War of 1991. Systematic Review. British Journal of Psychiatry. 2003;182:391-403

12. Pflanz S. Psychiatric Illness and the Workplace: Perspectives for Occupational Medicine in the Military. Militar Medicine. 1999;164(6):401-6.

13. Hull L, David A S, Hyams K C, Unwin C, Wessely S C, Hotopf M. Self-reported Health of Persian Gulf War Veterans: A Comparison of Help-seeking and Randomly Ascertained Cases. Military Medicine. 2002;167(9):747-52.

14. Thomas H V, Stimpson N J, Weightman A, Dunstan F, Lewis G. Pain in Veterans of the Gulf War of 1991: A Systematic Review. BMC Musculoskeletal Disorders. 2006;7:74.

15. Sharief M K, Priddin J, Delamont R S, Unwin C, Rose M R, David A, et al. Neurophysiologic Analysis of Neuromuscular Symptoms in UK Gulf War Veterans: A Controlled Study. Neurology. 2002;59(10):1518-25.

16. Ismail K, Kent K, Brugha T, Hotopf M, Hull L, Seed P, et al. The Mental Health of UK Gulf War Veterans: Phase 2 of a Two Phase Cohort Study. British Medical Journal. 2002;325(7364):576.

17. Iversen A C, Greenberg N. Mental Health of Regular and Reserve Military Veterans. Advances in Psychiatric Treatment. 2009; 15:100-6.

18. Ismail K, Blatchley N, Hotopf M, Hull L, Palmer I, Unwin C, et al. Occupational Risk Factors for Ill Health in Gulf Veterans of the United Kingdom. Journal of Epidemiology and Community Health. 2000;54(11):834-8.

19. Iversen A, Nikolaou V, Greenberg N, Unwin C, Hull L, Hotopf $\mathrm{M}$, et al. What Happens to British Veterans when They Leave the Armed Forces? European JournaL of Public Health. 2005;15(2):175-84.

20. Maas I, Settersten R A. Military Service During Wartime Effects on Men's Occupational Trajectories and Later Economic Well-Being. European Sociological Review. 1999;15:213-32.

21. O'Toole B I, Catts S V. Trauma, PTSD, and Physical Health: An Epidemiological Study of Australian Vietnam Veterans. Journal of Psychosomatic Research. 2008;64(1):33-40. 
22. O'Toole B I, Catts S V, Outram S, Pierse K R, Cockburn J. The Physical and Mental Health Of Australian Vietnam Veterans 3 Decades after the War and Its Relation to Military Service, Combat, and Post-Traumatic Stress Disorder. American Journal of Epidemiology. 2009;170(3):318-30.

23. Riddle J R, Smith T C, Smith B, Corbeil T E, Engel C C, Wells T S, et al. Millennium Cohort: The 2001-2003 Baseline Prevalence of Mental Disorders in the U.S. Military. Journal of Clinical Epidemiology. 2007;60(2):192-201.

24. Copley G B, Smith G S, Grayson J K, Gibson R L. Comparison of Two Sources of U.S. Air Force Injury Mortality Data. Military Medicine. 2003;168(10):792-6.

25. Pflanz S, Sonnek S. Work Stress in the Military: Prevalence, Causes, and Relationship to Emotional Health. Military Medicine. 2002;167(11):877-82.

26. Knezevic B, Belosevic L. Stress at Work among Military Doctors: A Preliminary Study. Acta Medica Croatica. 2006;60(4):309-14.

27. Smith T C, Smith B, Corbeil T E, Riddle J R, Ryan M A. Selfreported Mental Health among U.S. Military Personnel Prior and Subsequent to the Terrorist Attacks of September 11, 2001. Journal of Occupational and Environmental Medicine. 2004;46(8):775-82.

28. Smith T C, Zamorski M, Smith B, Riddle J R, Leardmann C A, Wells T S, et al. The Physical and Mental Health of a Large Military Cohort: Baseline Functional Health Status of the Millennium Cohort. BMC Public Health. 2007;7:340.

29. Robinson M E, Teyhen D S, Wu S S, Dugan J L, Wright A C, Childs J D, et al. Mental Health Symptoms in Combat Medic Training: A Longitudinal Examination. Military Medicine. 2009;174(6):572-7.

30. Sareen J, Cox B J, Afifi T O, Stein M B, Belik S L, Meadows G, et al. Combat and Peacekeeping Operations in Relation to Prevalence of Mental Disorders and Perceived Need for Mental Health Care: Findings from a Large Representative Sample of Military Personnel. Archives of General Psychiatry. 2007;64(7):843-52.

31. Lindstrom K E, Smith T C, Wells T S, Wang L Z, Smith B, Reed R J, et al. The Mental Health of U.S. Military Women in Combat Support Occupations. Journal of Women's Health (Larchmont N.Y.) 2006;15(2):162-72.

32. Rona R J, Hooper R, Jones M, Iversen A C, Hull L, Murphy D, et al. The Contribution of Prior Psychological Symptoms and Combat Exposure to Post Iraq Deployment Mental Health in the U.K Military. Journal of Traumatic Stress. 2009;22(1):119.

33. Hourani L L, Yuan H, Bray R M. Psychosocial and Health Correlates of Types of Traumatic Event Exposures among U.S Military Personnel. Military Medicine. 2003;168(9):736-43.

34. Solomon Z. The Impact of Posttraumatic Stress Disorder in Military Situations. Journal of Clinical Psychiatry. 2001;62(17):11-5

35. Rona R J, Jones M, Iversen A, Hull L, Greenberg N, Fear N T, et al. The Impact of Posttraumatic Stress Disorder on Impairment in the U.K. Military at the Time of the Iraq War. Journal of Psychiatric Research. 2009;43(6):649-55.
36. Bray R M, Fairbank J A, Marsden M E. Stress and Substance Use among Military Women and Men. American Journal of Drug and Alcohol Abuse. 1999;25(2):239-56.

37. Grayson J K, Gibson R L, Shanklin S L, Neuhauser K M, McGhee C. Trends in Positive Drug Tests, United States Air Force, Fiscal Years 1997-1999. Military Medicine. 2004;169(7):499504.

38. Hourani L L, Yuan H, Bray R M, Vincus A A. Psychosocial Correlates of Nicotine Dependence among Men and Women in the U.S. Naval Services. Addictive Behaviors. 1999;24(4):521-36.

39. Toledo D, González-Quevedo L A, Sanabria-Ferrand P A. Salud Mental y Consumo de Sustancias Psicoactivas en las Fuerzas Militares de Colombia. Universidad Militar Nueva Granada, editor. 1997. Ref Type: Unpublished Work.

40. González-Quevedo L A, Sanabria-Ferrand P A, Paredes O, Garcia M, Zuluaga H. Calidad de Vida y Salud Mental en la Fuerza Aérea Colombiana. Universidad Militar Nueva Granada, editor. 2007. Ref Type: Unpublished Work.

41. OMS. The World Health Organization Quality of Life assessment (WHOQOL): Position Paper from the World Health Organization. Social Science \& Medicine. 1995;41(10):1403-9.

42. Voelker M D, Saag K G, Schwartz D A, Chrischilles E, Clarke W R, Woolson R F, et al. Health-related Quality of Life in Gulf War Era Military Personnel. American Journal of Epidemiology. 2002;155(10):899-907.

43. Proctor S P, Harley R, Wolfe J, Heeren T, White R F. Healthrelated Quality of Life in Persian Gulf War Veterans. Military Medicine. 2001;166(6):510-9.

44. OMS. The World Health Organization Quality of Life Assessment (WHOQOL): Development and General Psychometric Properties. Social Science \& Medicine 1998;48(12):1569-85.

45. Seligman M E, Csikszentmihalyi M. Positive Psychology: An Introduction. American Psychologist 2000;55(1):5-14.

46. Aponte D M, Scoppetta O, González-Quevedo L A, SanabriaFerrand P A, Pineda N, Pardo C, et al. Condiciones Teórico Metodológicas para la Realización de un Diagnóstico Territorial de Salud Mental en Santafé de Bogotá. Investigaciones en Seguridad Social y Salud 2000;(2).

47. Ryff C D, Keyes C L. The Structure of Psychological Wellbeing Revisited. Journal of Personality and Social Psychology. 1995;69(4):719-27.

48. Tennant R, Hiller L, Fishwick R, Platt S, Joseph S, Weich S, et al. The Warwick-Edinburgh Mental Well-being Scale (WEMWBS): Development and U.K. Validation. Health and Quality of Life Outcomes. 2007;5(63).

49. Hambleton R, Swaminathan H. Item Response Theory. Principles and applications. Boston: Kluwer Nijhoff Publishing; 1985.

50. Toomey R, Kang H K, Karlinsky J, Baker D G, Vasterling J J, Alpern R, et al. Mental Health of U.S. Gulf War Veterans 10 Years After the War. British Journal of Psychiatry. 2007; 190:385-93.

51. The Iowa Persian Gulf Study Group. Self-reported Illness and Health Status among Gulf War Veterans. A Population-based Study. JAMA. 1997;277(3):238-45. 